\title{
Measurements of Hadron Production in Pion-Carbon Interactions with NA61/SHINE at the CERN SPS
}

\author{
Raul R. Prado*1,2 for the NA61/SHINE Collaboration ${ }^{\dagger}$ \\ ${ }^{1}$ IKP, Karlsruhe Institute of Technology (KIT), Postfach 3640, D-76021 Karlsruhe, Germany \\ ${ }^{2}$ Instituto de Física de São Carlos, Universidade de São Paulo, Brazil \\ E-mail: raul.prado@usp.br
}

\begin{abstract}
NA61/SHINE is a fixed target experiment designed to study hadron-proton, hadron-nucleus and nucleus-nucleus interactions at the CERN Super-Proton-Synchrotron. In this proceeding we present results on spectra of identified hadrons produced in pion-carbon production interactions, which are of fundamental importance to improve the extensive air shower modeling, and hence the interpretation of ultra-high-energy-cosmic-rays measurements. In particular, our measurements of (anti)baryons and $\rho^{0}$ production in pion-carbon interactions can improve the predictions of muon production by air shower simulations using hadronic interaction models. In this contribution we discuss the data analysis and the results from the analysis of pion-carbon collisions recorded at beam momenta of 158 and $350 \mathrm{GeV} / c$. The preliminary spectra of $\mathrm{K}^{ \pm}$and $\mathrm{p}(\overline{\mathrm{p}})$ are shown, as well as a comparison to predictions of hadronic interaction models used in air shower simulations. Additionally, we present final results on the production of $\rho^{0}, \omega$ and $\mathrm{K}^{* 0}$ resonances.
\end{abstract}

35th International Cosmic Ray Conference - ICRC2017

10-20 July, 2017

Bexco, Busan, Korea

\footnotetext{
${ }^{*}$ Speaker.

${ }^{\dagger}$ http://shine.web.cern.ch/content/author-list
} 


\section{Introduction}

Indirect measurements of high energy cosmic rays through Extensive Air Showers (EAS) generally require air shower simulations to be interpreted. These simulations are performed by Monte Carlo codes that make use of hadronic interaction models to describe the nucleus-air and hadron-air collisions along the shower development [1]. During the last decades, a number of EAS experiments (like HiRes-MIA [2,3], KASCADE-Grande [4] and Pierre Auger Observatory [5]) have reported results indicating that simulations do not satisfactorily predict the muon production in air showers [6-10]. This discrepancy between data and simulations has strong implications mainly on inferences of the cosmic rays mass composition [11].

The production of (anti)baryon and $\rho^{0}$ along the air shower development have been shown to be very important mechanisms for the muon production [12-14]. Therefore, a proper prediction of these particles spectra by the hadronic interaction models could reduce the discrepancy between air shower simulations and data. Although the current hadronic models are tuned by making use of a large body of proton-nucleus data, there is a lack of measurements of particle production in $\pi$-nucleus interactions, which are the most abundant hadronic interaction occurring in an EAS.

To address the lack of suitable data for the tuning of hadronic interaction models used in air shower simulations, the NA61/SHINE experiment [15] collected new data with negatively charged pion beams at 158 and $350 \mathrm{GeV} / c$ incident on a thin carbon target. A selection of results on the hadron and meson productions of these collisions are presented in this proceeding.

\section{The NA61/SHINE experiment}

NA61/SHINE (SHINE = SPS Heavy Ion and Neutrino Experiment) is a fixed target experiment at the CERN SPS designed to study hadron production in nucleus-nucleus and hadron-nucleus collisions. Its physics goals comprise a) the strong interaction program, which investigates the properties of the onset of deconfinement and search for the critical point of strongly interacting matter, $b$ ) the neutrino program, to precisely measure the hadron production important to calculate the neutrinos and antineutrino fluxes in the T2K neutrino experiment [16], and c) the cosmic rays program, focused on the measurements of the hadron and meson production which are most relevant for the modeling of extensive air showers. The full description of the NA61/SHINE experiment and its science program can be found in Ref. [15]

The NA61/SHINE detector measures charged particles produced by the collision of the beam particles with the target through a set of five Time Projection Chambers (TPC). Since two of the TPCs are placed in the magnetic field produced by superconducting dipole magnets, the charge and the momenta of the particles can be measured and the achieved resolution on $p$ is of the order of $\sigma(p) / p^{2}=10^{-4}(\mathrm{GeV} / c)^{-1}$. Additionally, the energy loss per unit of length $(\mathrm{d} E / \mathrm{d} x)$ in the TPCs is used in this work for particle identification.

A beam detector system composed of scintillation and Cherenkov counters is placed upstream of the detector to identify and measure the beam particles. The position of the beam is measured by a set of three beam position detectors, which are also placed upstream of the target.

The data analyzed in this work are collisions of a $\pi^{-}$secondary beam with a thin $(2 \mathrm{~cm})$ carbon target. Two data sets were recorded in 2009 with beam energies of 158 and $350 \mathrm{GeV} / c$. 


\section{3. $\pi^{ \pm}, K^{ \pm}, \mathbf{p}$ and $\overline{\mathbf{p}}$ spectra}

In NA61/SHINE, charged particles can be identified via the track-by-track measurement of the truncated mean of the deposited energy registered by individual pads in the TPCs.

After splitting the data into bins of total and transverse momentum ( $p$ and $p_{\mathrm{T}}$ ), the measured $\mathrm{d} E / \mathrm{d} x$ distributions are fitted by a generic $\mathrm{d} E / \mathrm{d} x$ model that combines the contributions of 5 particle types ( $e, \pi, K, p$ and deuterons). The $\mathrm{d} E / \mathrm{d} x$ distributions for individual particles are described by the model as asymmetric Gaussians in which the resolution $\sigma$ depends on the number of clusters $N_{\mathrm{cl}}$ in each track as $\sigma \sim 1 / \sqrt{N_{\mathrm{cl}}}$. The particle yields are then obtained by integrating the fitted distributions.

The fits of the $\mathrm{d} E / \mathrm{d} x$ distributions provide a precise particle identification for most of the phase space bins. However, for a number of bins, mainly in the low momentum region, the average energy deposit for different particle types can be very similar, which implies a large overlap between the two $\mathrm{d} E / \mathrm{d} x$ distributions. For these bins, the yields of the overlapping particles cannot be precisely determined by the $\mathrm{d} E / \mathrm{d} x$ fit method and therefore they must be excluded from the particle spectra. To determine which bins to exclude, a large set of simulations of the $\mathrm{d} E / \mathrm{d} x$ distributions were produced, reproducing the same $p, p_{\mathrm{T}}$ and $N_{\mathrm{cl}}$ distributions and similar particle yields observed in data. After performing the $\mathrm{d} E / \mathrm{d} x$ fit procedure on the simulated data set, the standard deviation and the average bias of the yields were computed, and the bins with a relative standard deviation larger than $25 \%$ for a given particle were excluded from the analysis. For the remaining bins, the average biases were corrected and the statistical uncertainties were computed by the standard deviation of the simulated data fits.

The particle yields obtained from the $\mathrm{d} E / \mathrm{d} x$ distribution fits were then corrected for detector effects (acceptance and selection efficiency) and feed-down contributions from weak decays and re-interactions in the target and downstream detector material. Simulated data using three hadronic interaction models (EPOS 1.99 [12], QGSJeT II-04 [17] and DPMJET 3.06 [18]) was used to compute the correction factors. While the correction factor dependence on the specific hadronic model was observed to be $<2 \%$ for the acceptance and selection efficiency, the differences between the feed-down contribution can reach up to $10 \%$, depending on the $p$ and $p_{\mathrm{T}}$ bin and particle type. These differences were accounted for in the systematic uncertainties of the final spectra.

Finally, the average particle multiplicity for each $p$ and $p_{\mathrm{T}}$ bin was computed by dividing the corrected yields by the total number of target interactions, which in turn was obtained by correcting the total number of triggered events by the trigger and event-selection efficiency. This correction was also computed by using simulations with the aforementioned hadronic interaction models and amounts to $92.5 \pm 3.5 \%$ and $78.5 \pm 4.0 \%$ for the 158 and $350 \mathrm{GeV} / c$ data set, respectively, where the uncertainties were estimated by the differences between the factor obtained with different hadronic models. The final spectra of $\mathrm{K}^{ \pm}$and $\mathrm{p}(\overline{\mathrm{p}})$ are shown in Fig. 1 . The preliminary $\pi^{ \pm}$spectra can be found in Ref. [19].

For each $p$ bin, it was verified that the average multiplicity as a function of $p_{\mathrm{T}}$ can be well described by an exponential function in $m_{\mathrm{T}}$, which means $\frac{d n}{d p_{\mathrm{T}}} \sim p_{\mathrm{T}} \exp \left[-\left(p_{\mathrm{T}}^{2}+m_{0}^{2}\right)^{\frac{1}{2}} / T\right]$, with particle mass $m_{0}$ and inverse slope parameter $T$. This function was fitted to the data and the result is shown as dashed lines in Fig. 1.

The integrated spectra over $p_{\mathrm{T}}$ for $\pi^{ \pm}, \mathrm{K}^{ \pm}$and $\mathrm{p}(\overline{\mathrm{p}})$ are shown in Figs. 2 and 3, where they are 


\section{NA61/SHINE preliminary}

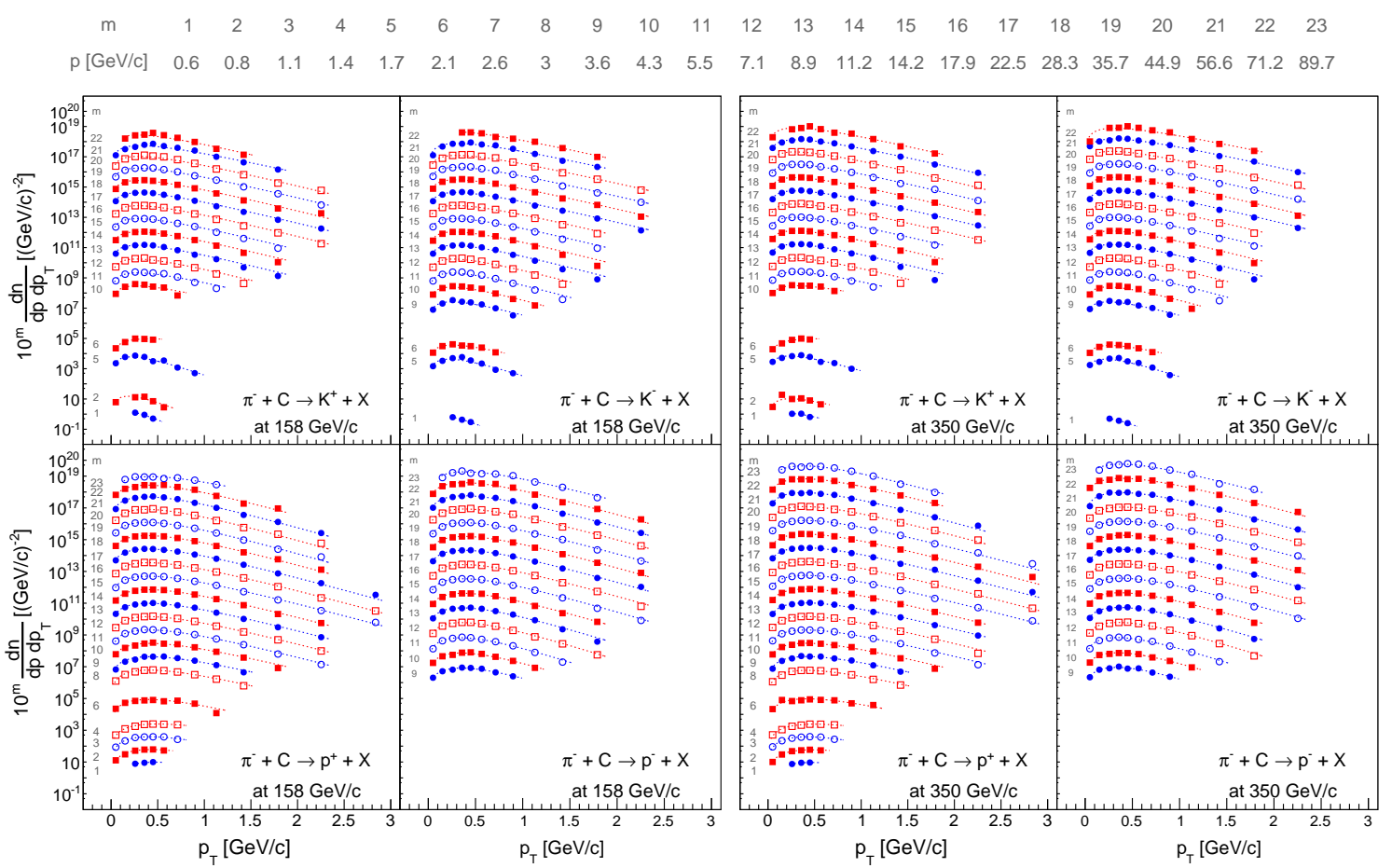

Figure 1: Spectra of $\mathrm{K}^{ \pm}$and $\mathrm{p}(\overline{\mathrm{p}})$ as function of $p_{\mathrm{T}}$ at $158 \mathrm{GeV} / c$ (left) and $350 \mathrm{GeV} / c$ (right). For each $p$ the spectrum was multiplied by $10^{m}$ with the value of $m$ shown on the left.

compared to the predictions of EPOS 1.99, DPMJET 3.06, SiBYLL 2.3C [20], QGSJET II-04 and Epos LHC [21]. Note that due to an improved subtraction of non-target interactions, the updated $\pi^{ \pm}$spectra shown here differ from the preliminary ones presented in Ref. [19] by $\leq 10 \%$. To perform the integration, two functions were used to extrapolate the $p_{\mathrm{T}}$ range which is not covered by the measured spectra: an exponential in $m_{\mathrm{T}}$ and a Gaussian convoluted with an exponential. The average spectra obtained by using these functions are shown in Figs. 2 and 3 and the difference between both was added to the systematic uncertainties, being in all cases $<1 \%$. The $p$ bins in which the contribution from the extrapolation is larger than $5 \%$ are not shown.

\section{4. $\rho^{0}, \omega$ and $\mathbf{K}^{* 0}$ spectra}

The yields of the resonance mesons can be measured using the NA61/SHINE apparatus through the inclusive $\pi^{+} \pi^{-}$invariant mass spectra. The resonance signal can be extracted by fitting templates of the $\pi^{+} \pi^{-}$invariant mass distribution, obtained from Monte Carlo simulation, to the measured distribution. The Monte Carlo events were generated using EPOS 1.99 as hadronic interaction model and they were passed through the full NA61/SHINE detector simulation and reconstruction chain. To generate the template for the combinatorial background, two approaches were applied: the charge mixing method, in which the $\pi^{+} \pi^{+}$and $\pi^{-} \pi^{-}$are treated as the background, and the Monte Carlo method, in which the background mass distribution is obtained directly from simulations. 

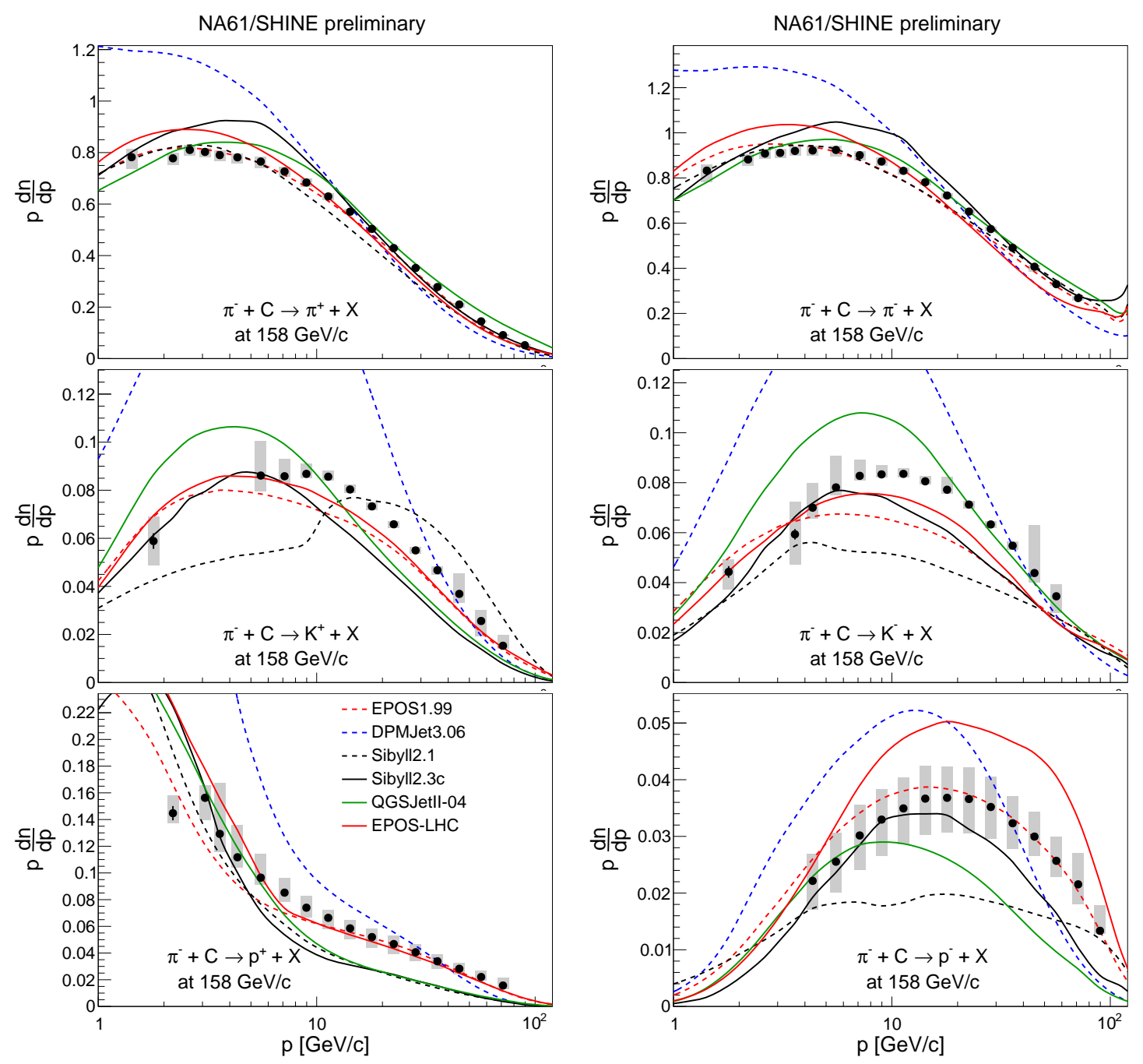

Figure 2: Spectra of $\pi^{ \pm}, \mathrm{K}^{ \pm}$and $\mathrm{p}(\overline{\mathrm{p}})$ as a function of $p$, integrated over $p_{\mathrm{T}}$, for the $158 \mathrm{GeV} / c$ data set. The statistical uncertainties are represented by bars and the systematic ones by gray bands.

The fitted yields were then corrected for the limited detector acceptance, selection efficiency, detector trigger efficiency, fitting bias and feed-down from re-interactions. Simulations using EPOS 1.99 were used to compute the corresponding correction factors. Finally, the average multiplicity was obtained by dividing the corrected yields by the total number of target interactions. The complete analysis description and results can be found in Ref. [22].

In Fig. 4 , the spectra of $\rho^{0}, \omega$ and $\mathrm{K}^{* 0}$ are shown and compared to the predictions of EPOS 1.99, DPMJet 3.06, Sibyll 2.1, Sib YLl 2.3 [23], QGSJet II-04 and EPOS LHC. While the $\rho^{0}$ spectra are shown for beam energies of 158 and $350 \mathrm{GeV} / c$, the $\omega$ and $\mathrm{K}^{* 0}$ spectra are limited to the $158 \mathrm{GeV} / c$ data set because of the large uncertainties obtained at $350 \mathrm{GeV} / c$. Additionally, the $\rho^{0}$ spectrum at $350 \mathrm{GeV} / c$ is limited to $x_{\mathrm{F}}<0.5$ because of the limited acceptance of the detector at this beam energy.

The systematic uncertainties on the spectra were estimated by taking into account the contri- 

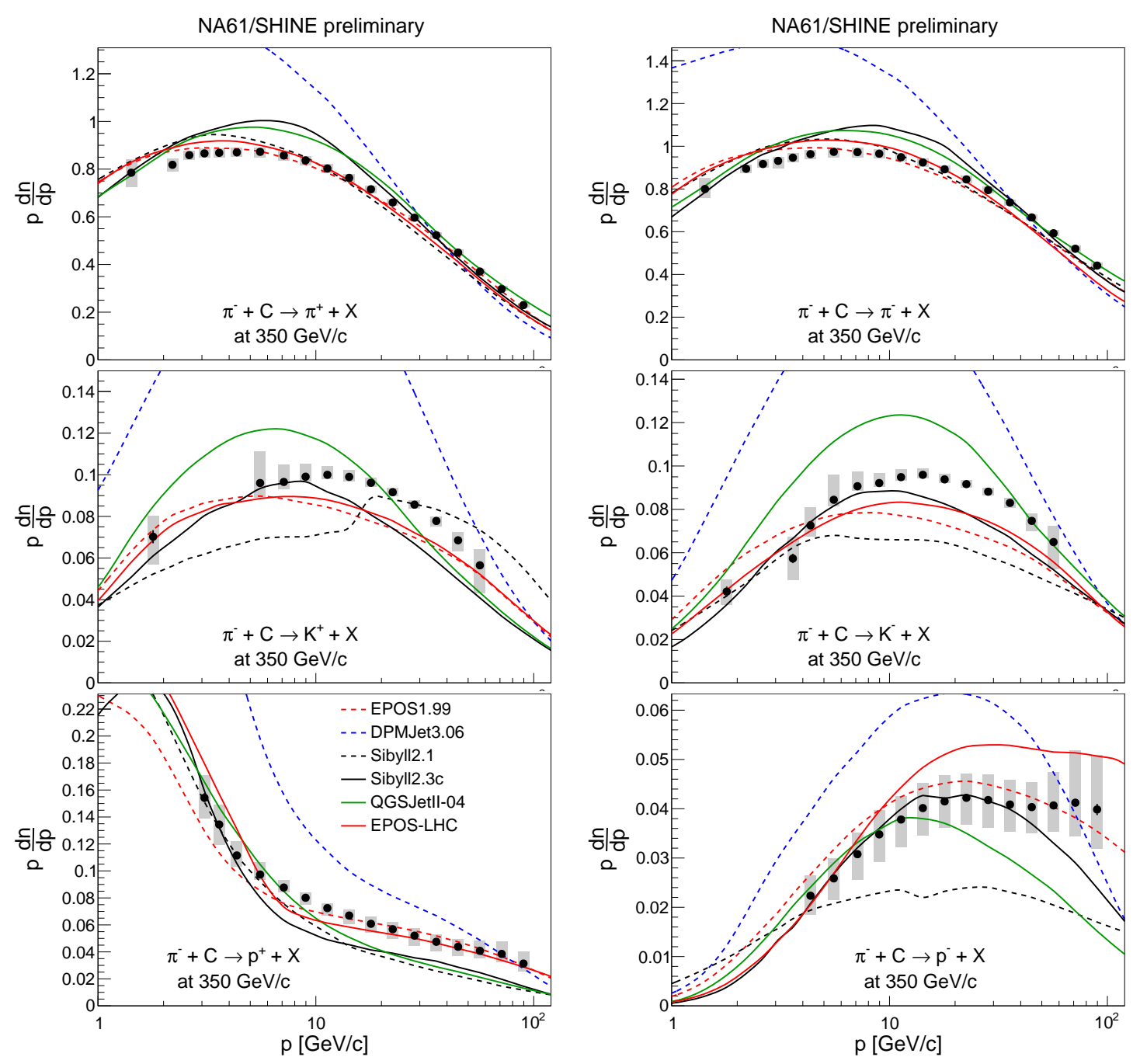

Figure 3: Spectra of $\pi^{ \pm}, \mathrm{K}^{ \pm}$and $\mathrm{p}(\overline{\mathrm{p}})$ as a function of $p$, integrated over $p_{\mathrm{T}}$, for the $350 \mathrm{GeV} / c$ data set. The statistical uncertainties are represented by bars and the systematic ones by gray bands.

butions of the difference between two methods to determine the combinatorial background, differences on the correction factors computed with different hadronic interaction models and differences due to variations on the track and event selection criteria.

\section{Summary and conclusions}

Selected results of particle production in pion-carbon collisions measured by NA61/SHINE experiment were presented in this proceeding. First we have shown the spectra of $\pi^{ \pm}, K^{ \pm}$and $p$ $(\overline{\mathrm{p}})$, obtained by means of a particle identification analysis based on the $\mathrm{d} E / \mathrm{d} x$. Second, we have shown the spectra of $\rho^{0}, \omega$ and $\mathrm{K}^{* 0}$, obtained by means of a template fit method applied to the invariant mass distribution of $\pi^{+} \pi^{-}$. The most relevant sources of systematic uncertainties were estimated and presented with the final spectra. 

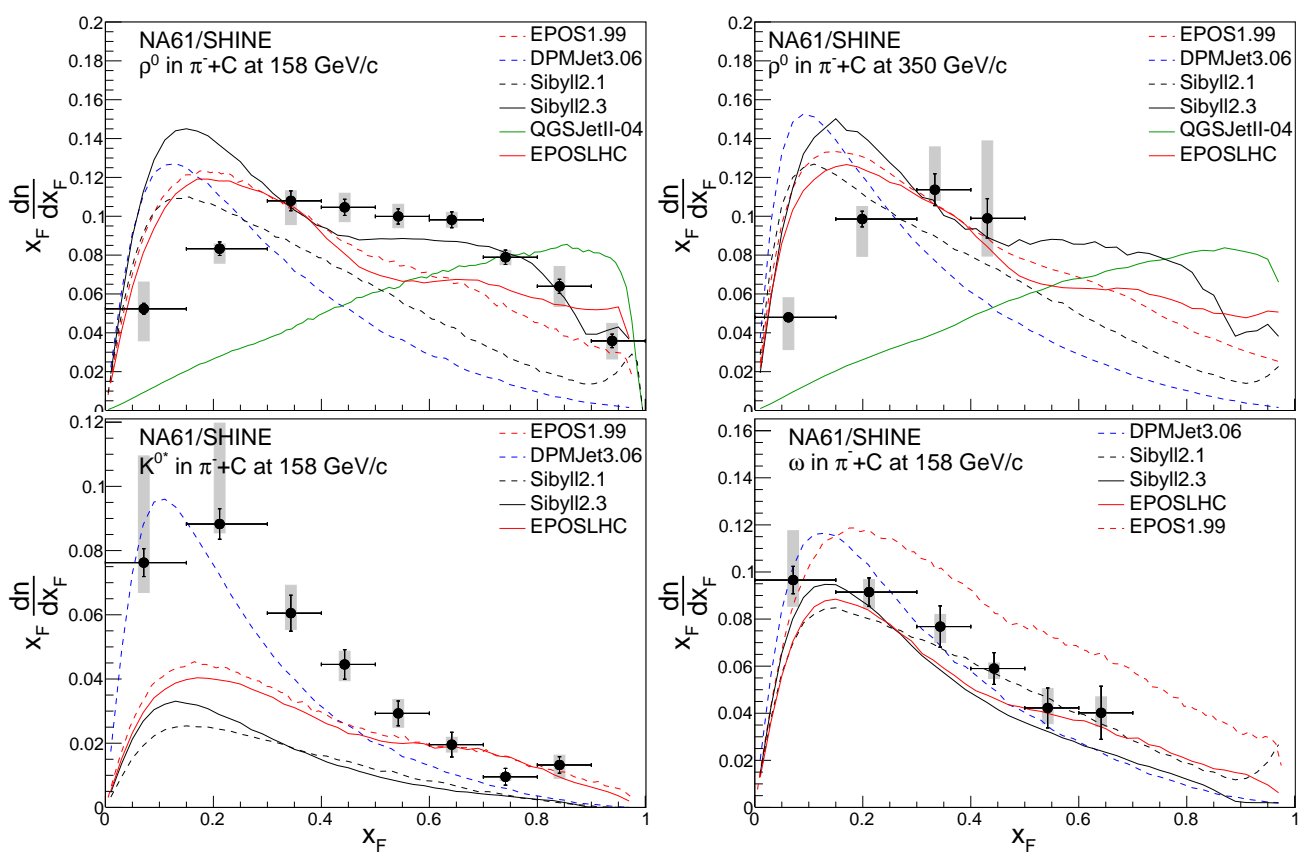

Figure 4: Spectra of $\rho^{0}, \omega$ and $\mathrm{K}^{* 0}$ as a function of $x_{\mathrm{F}}$. The statistical uncertainties are represented by bars and the systematic ones by gray bands [22].

From the comparison of the measured $\pi^{ \pm}, \mathrm{K}^{ \pm}$and $\mathrm{p}(\overline{\mathrm{p}})$ spectra, one can see that none of the models provide a satisfactory description of all particle production, at both energies. In the special case of $\bar{p}$, which is important for air shower modeling, EPOS 1.99 provides the best agreement, while its production is overestimated by EPOS LHC and DPMJET 3.06, and underestimated by QGSJET II-04 and SIBYLL 2.1. Concerning the spectra of $\rho^{0}$, an overall overestimation of its production by the models is observed at $x_{\mathrm{F}}<0.3$, while at $x_{\mathrm{F}}>0.3$, an overall underestimation is observed instead.

\section{Acknowledgments}

We would like to thank the CERN EP, BE and EN Departments for the strong support of NA61/SHINE.

This work was supported by the Hungarian Scientific Research Fund (grants OTKA 68506 and 71989), the János Bolyai Research Scholarship of the Hungarian Academy of Sciences, the Polish Ministry of Science and Higher Education (grants 667/N-CERN/2010/0, NN 202484339 and NN 20223 1837), the Polish National Center for Science (grants 2011/03/N/ST2/03691, 2013/11/N/ST2/03879, 2014/13/N/ST2/02565, 2014/14/E/ST2/00018 and 2015/18/M/ST2/00125, 2015/19/N/ST2 /01689), the Foundation for Polish Science - MPD program, co-financed by the European Union within the European Regional Development Fund, the Federal Agency of Education of the Ministry of Education and Science of the Russian Federation (SPbSU research grant 11.38.242.2015), the Russian Academy of Science and the Russian Foundation for Basic Research (grants 08-02-00018, 09-02-00664 and 12-02-91503-CERN), the National Research Nuclear University MEPhI in the framework of the Russian Academic Excellence Project (contract No. 02.a03.21.0005, 27.08.2013), the Ministry of Education, Culture, Sports, Science and Technology, Japan, Grant-in-Aid for Scientific Research (grants 18071005, 19034011, 19740162, 20740160 and 20039012), the German Research Foundation (grant GA 1480/2-2), the EU-funded Marie Curie Outgoing Fellowship, Grant PIOF-GA-2013-624803, the Bulgarian Nuclear Regulatory Agency and the Joint Institute for Nuclear 
Research, Dubna (bilateral contract No. 4418-1-15/17), Bulgarian National Science Fund (grant DN08/11), Ministry of Education and Science of the Republic of Serbia (grant OI171002), Swiss Nationalfonds Foundation (grant 200020117913/1), ETH Research Grant TH-01 07-3 and the U.S. Department of Energy.

RRP would like to thank the financial support by Fundação de Amparo à Pesquisa do Estado de São Paulo (FAPESP, proc. 2016/12735-3).

\section{References}

[1] R. Engel, D. Heck, and T. Pierog Annu. Rev. Nucl. Part. Sci. 61 (2011) 467-489.

[2] T. Abu-Zayyad et al. Nucl. Instrum. Meth. A 450 (1999) 253-269.

[3] A. Barione et al. Nucl. Instrum. Meth. A 346 (1994) 329-352.

[4] G. Navarra et al. [KASCADE-Grande Collab.] Nucl. Instrum. Meth. A 518 (2004) 207-209.

[5] A. Aab et al. [Pierre Auger Collab.] Nucl. Instrum. Meth. A 798 (2015) 172-213. [arXiv:1502.01323].

[6] T. Abu-Zayyad et al. Phys. Rev. Lett. 84 (2000) 4276-4279.

[7] J. C. Arteaga-Velazquez et al. EPJ Web Conf. 52 (2013) 07002.

[8] A. Aab et al. [Pierre Auger Collab.] Phys. Rev. D 90 (2014) 012012. [arXiv: 1407.5919 ]

[9] A. Aab et al. [Pierre Auger Collab.] Phys. Rev. D 91 (2015) 032003. [arXiv: 1408 . 1421 ] .

[10] A. Aab et al. [Pierre Auger Collab.] Phys. Rev. Lett. 117 (2016) 192001. [arXiv: 1610 . 08509 ]

[11] K.-H. Kampert and M. Unger Astropart. Phys. 35 (2012) 660-678. [arXiv: 1201.0018 ] .

[12] T. Pierog and K. Werner Phys. Rev. Lett. 101 (2008) 171101. [arXiv:astro-ph/0611311].

[13] H.-J. Drescher Phys. Rev. D 77 (2008) 056003. [arXiv: 0712.1517 ].

[14] S. Ostapchenko EPJ Web Conf. 52 (2013) 02001.

[15] N. Abgrall et al. [NA61/SHINE Collab.] JINST 9 (2014) P06005. [arXiv: 1401.4699 ] .

[16] K. Abe et al. [T2K Collab.] Nucl. Instrum. Meth. A 659 (2011) 106-135. [arXiv:1106.1238] .

[17] S. Ostapchenko Phys. Rev. D 83 (2011) 014018. [arXiv:1010.1869].

[18] S. Roesler, R. Engel and J. Ranft, The Monte Carlo Event Generator DPMJET-III, pp. 1033-1038. Springer Berlin Heidelberg, Berlin, Heidelberg, 2001.

[19] A. Herve [NA61/SHINE Collab.] PoS ICRC2015 (2015) 330. [arXiv: 1509 . 06586 ] .

[20] R. Engel et al. PoS ICRC2017 (2017) 349.

[21] T. Pierog et al. Phys. Rev. C 92 (2015) 034906. [arXiv:1306.0121].

[22] A. Aduszkiewicz et al. [NA61/SHINE Collab.]. Submitted to Eur. Phys. J. C [arXiv:1705.08206].

[23] F. Riehn et al. PoS ICRC2015 (2015) 558. [arXiv:1510. 00568 ]. 